\title{
The Combined Use of 5 or More Drugs Is a Factor Related to Lower Adherence to S-1 in S-1 and Oxaliplatin Treatment for Advanced Gastric Cancer
}

\author{
Kazuyoshi Kawakami, ${ }^{* a}$ Takeshi Aoyama, ${ }^{a}$ Takashi Yokokawa, ${ }^{a}$ Kazuo Kobayashi, ${ }^{a}$ \\ Daisuke Takahari, ${ }^{b}$ Keisho Chin, ${ }^{b}$ Tsugumi Ide ${ }^{c}$ Yoshiaki Machida, ${ }^{c}$ Kensei Yamaguchi, ${ }^{b}$ and \\ Toshihiro Hama ${ }^{a}$ \\ ${ }^{a}$ Department of Pharmacy, Cancer Institute Hospital, Japanese Foundation for Cancer Research; 3-10-6 Ariake, \\ Koto-ku, Tokyo 135-8550, Japan: ${ }^{b}$ Department of Gastroenterological Chemotherapy, Cancer Institute Hospital, \\ Japanese Foundation for Cancer Research; 3-8-31 Ariake, Koto-ku, Tokyo 135-8550, Japan: and ${ }^{c}$ Section for \\ Practical Education, Hoshi University School of Pharmacy and Pharmaceutical Sciences; 2-4-41 Ebara, \\ Shinagawa-ku, Tokyo 142-8501, Japan. \\ Received February 27, 2021; accepted May 21, 2021
}

S-1 plus oxaliplatin (SOX) is an established treatment for advanced gastric cancer. S-1 adherence is the key to successful SOX treatment. This study focused on S-1 adherence by evaluating real-world adherence to S-1 and investigating factors related to decreased S-1 adherence. This study included cases treated between August 1, 2014 and October 12, 2016 at the Cancer Institute Hospital of the Japanese Foundation for Cancer Research. The S-1 adherence rate per cycle was defined as the number of times a patient took S-1/28. In this study, adherence to S-1 was assessed through pill counts and by asking the patient about the reason for nonadherence at a pharmaceutical outpatient clinic. Univariate and multivariate analyses were performed to investigate factors influencing lower adherence. This analysis included 116 patients evaluated for adherence to $\mathrm{S}-1$ on SOX treatment. The median rate of adherence to $\mathrm{S}-1$ was $92.8 \%$ in the first cycle and $90.5 \%$ in the seventh cycle. The median relative dose intensity of $\mathrm{S}-1$ was $84.6 \%$. In terms of reasons for nonadherence, patients most commonly cited nausea/vomiting (43.7\%), diarrhea $(20.8 \%)$, missed dose (11.8\%), and fever (8.1\%). Logistic regression analysis was performed using the most appropriate regression equation, and a significant association was detected with 1 factor, number of combined drugs $\geq 5$ (odds ratio $(\mathrm{OR})=2.50$; 95\% confidence interval (CI), 1.04-6.03, $p=0.04)$. Eliminating unnecessary concomitant medications helps maintain proper adherence to $\mathrm{S}-1$ in SOX treatment.

Key words S-1; S-1 plus oxaliplatin (SOX); adherence; pharmaceutical outpatient clinic; oral anticancer drug

\section{INTRODUCTION}

Gastric cancer is the third most common cause of cancerrelated deaths globally, with the highest estimated mortality in East Asia. ${ }^{1)}$ Standard treatment for advanced gastric cancer has been established. The usefulness of cisplatin plus S-1, capecitabine plus oxalipatin (CapOx) ${ }^{3)}$ and S-1 plus oxaliplatin $(\mathrm{SOX})^{4)}$ has been established for metastatic gastric cancer, and they are used in clinical settings. The standard treatment for gastric cancer has also been established, and to maximize the therapeutic effect, the established standard treatment should be performed while maintaining the patient's QOL in the real world.

In gastric cancer, S-1 and capecitabine are incorporated into standard therapy, and oral anticancer drug adherence becomes important. S-1 is an oral anticancer preparation consisting of tegafur, a prodrug of fluorouracil (5-FU), and the modulators gimeracil and oteracil potassium. Gimeracil blocks the degradation of 5-FU by the reversible inhibition of dihydropyrimidine dehydrogenase, which is the primary enzyme metabolizing fluorouracil. Oteracil potassium is distributed in the gastrointestinal tract at a high concentration following oral administration, and it prevents phosphorylation (i.e., activation) of 5-FU by inhibiting the effect of orotate phosphoribosyl transferase $e^{5,6)}$ Adherence to treatment with oral anticancer agents (imatinib, sunitinib, etc.) and factors affecting adherence have been assessed.7) In other words, with appropriate supportive care, oral anticancer drugs can be taken internally. Therefore, strategies for improving adherence to oral anticancer drugs can be developed by evaluating adherence to oral anticancer drugs, clarifying the reasons for nonadherence, and taking countermeasures against them. For gastric cancer in particular, there are cases where oral drugs cannot be taken due to the underlying disease, and it is necessary to improve adherence to oral anticancer drugs to enhance the therapeutic effect. However, in clinical practice, adherence to oral anticancer drugs is often not evaluated.

Since no gold standard has been established for the evaluation of adherence to oral anticancer drugs, ${ }^{8)}$ there are various methods for evaluating adherence. Certain methods for measuring adherence are preferred in specific clinical or research settings, and a combination of measures maximizes accuracy. ${ }^{9)}$ In this study, adherence was evaluated using a method that combines pill counts, which is an objective evaluation, and self-report, which is one of the direct methods. This study determined adherence to $\mathrm{S}-1$ and the factors that reduce adherence to S-1. Useful information on the management of SOX treatment for gastric cancer is presented. 


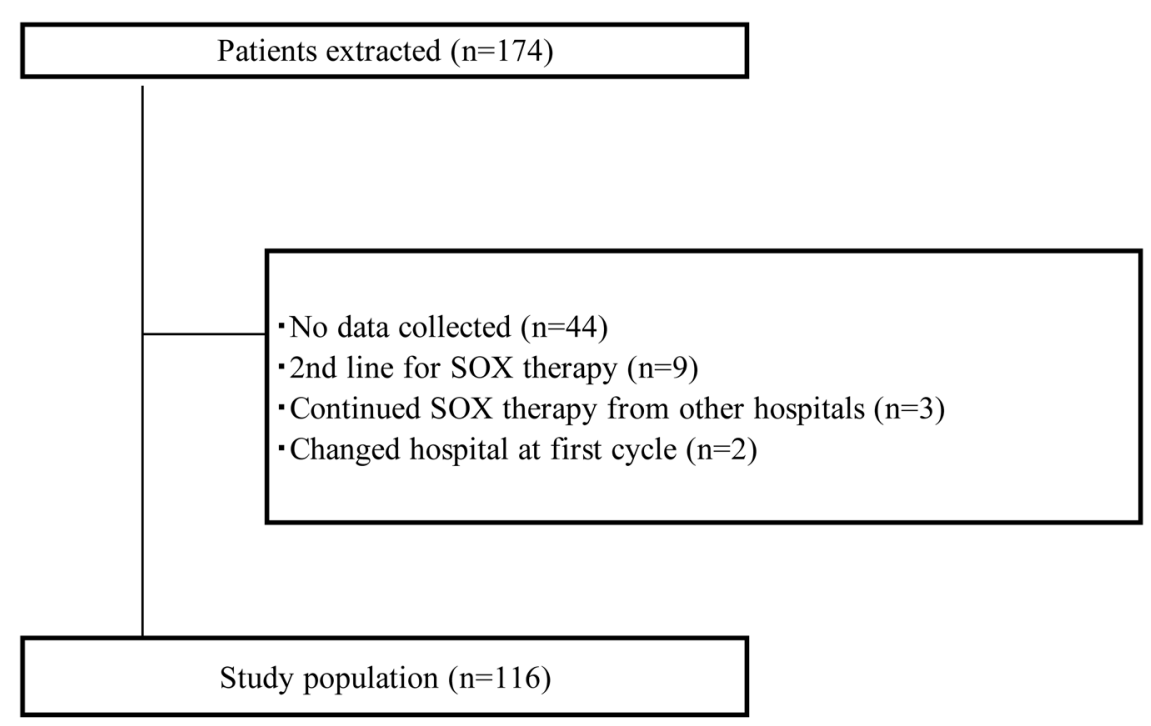

Fig. 1. Patient Flow Chart and Reasons for Exclusion

A total of 174 patients at the Cancer Institute Hospital of the Japanese Foundation for Cancer Research were identified. SOX: S-1 plus oxaliplatin.

\section{PATIENTS AND METHODS}

Study Design and Treatment Data of 174 consecutive patients who received SOX treatment for advanced gastric cancer between August 1, 2014 and October 12, 2016 at the Cancer Institute Hospital of the Japanese Foundation for Cancer Research were reviewed. In SOX treatment, S-1 was given orally at $80 \mathrm{mg} / \mathrm{m}^{2}$ twice a day for $14 \mathrm{~d}$, followed by a 7-d rest period. On day 1 , oxalipratin $\left(130 \mathrm{mg} / \mathrm{m}^{2}\right)$ was administered intravenously. Trastuzumab was administered intravenously at a dose of $8 \mathrm{mg} / \mathrm{kg}$ for the first cycle and $6 \mathrm{mg} / \mathrm{kg}$ for the subsequent cycles on day 1 . Courses of treatment were repeated every $21 \mathrm{~d}$ until disease progression or unacceptable toxicity.

As preventive measures against nausea and vomiting, palonosetron $0.75 \mathrm{mg}$ on day 1 in addition to oral aprepitant (125 mg on day 1 and $80 \mathrm{mg} / \mathrm{d}$ on days $2-3$ ) and intravenous dexamethasone $6.6 \mathrm{mg} / \mathrm{d}$ on day 1 followed by oral dexamethasone $4 \mathrm{mg} / \mathrm{d}$ on days $2-4$ were given. For diarrhea, loperamide $1 \mathrm{mg}$ was given at the time of diarrhea onset. As preventive measures against a hand-foot skin reaction, heparinoid ointment was applied to the hands and feet.

The S-1 adherence rate in a cycle was defined as the number of times a patient took S-1 in a 14-d cycle divided by the prescribed 28 doses. Relative dose intensities and factors associated with influencing lower adherence to S-1 were determined retrospectively from electronic records. Non-adherence defined as not taking medication in this study. ${ }^{10)}$

Data Collection Adherence to S-1 was assessed using the pill count and patients' treatment journals, and non-adherent patients were interviewed to ask about their reasons for not taking S-1 at every visit to a pharmaceutical outpatient clinic. If there are multiple reasons, we asked for the reason that had the strongest impact. To determine the pill count, any unused pills were counted during visits to a pharmaceutical outpatient clinic. If the patient had not brought remaining unused S-1 pills, a pill count could not be performed. All participants in this study visited a pharmaceutical outpatient clinic and completed the assessments.

The median relative dose intensities ${ }^{11)}$ of S-1 and factors influencing lower a adherence were retrospectively determined using electronic patient records. This study protocol was approved by the clinical research ethics review committee of the Cancer Institute Hospital of the Japanese Foundation for Cancer Research (Approval No. 2015-1118).

Pharmaceutical Outpatient Clinic In the Pharmaceutical Outpatient Clinic of the Cancer Institute Hospital of the Japanese Foundation for Cancer Research, pharmacists checked adherence to oral anticancer drugs and assessed side effects, which could be one cause of non-adherence, before the patients were examined by their physicians. ${ }^{12,13)}$ In particular, the pharmacists played two important roles, confirmation and suggestion. Confirmation involved checking the patients' adherence to S-1 and evaluating the side effects. Suggestion involved including in patients' electronic medical records suggestions regarding the most effective prescription for supportive pharmacotherapy, the timing of the next anticancer drug dose, and the administration regimen.

Statistical Analysis This analysis was based on 116 patients evaluated for adherence to $\mathrm{S}-1$. The outcome variable was adherence, dichotomized according to success, which defined as adherence rate $<95 \%$ in the first cycle. Univariate analyses were then performed using sex, age ( $\geq 70$ years vs. $<70$ years), ECOG PS (2 vs. 0, 1), living status (living alone $v s$. with family), creatinine clearance $(\leq 60 \mathrm{~mL} / \mathrm{min} v s$. $>60 \mathrm{~mL} / \mathrm{min}$ ), number of combined drugs before the start of SOX treatment ( $\geq 5$ vs. 0-4), married (vs. unmarried), total gastrectomy (yes vs. no), disease status, $\mathrm{Alb}(<3 \mathrm{~g} / \mathrm{dL} v s$. $\geq 3 \mathrm{~g} / \mathrm{dL}$ ), trastuzumab, and oxaliplatin dose (first cycle). Regarding age, previous studies comparing age with adherence to oral anticancer drugs have been reported. ${ }^{14,15)}$ The study examined people aged 70 years and over and 80 years and over. In this study, it was judged that the ratio of people aged 80 years and over was small, and a comparative study was conducted based on the age of 70 years and over. The number of combined drugs, it has been reported that the frequency of adverse drug events increases with 6 or more types of oral medications. ${ }^{16)}$ Regarding albumin, National Cancer InstituteCommon Terminology Criteria for Adverse Events ver5.0 hypoalbuminemia Grade 2 or higher is albumin $3 \mathrm{~g} / \mathrm{dL}$ or lower. In patients has Grade 2 hypoalbuminemia, the general 
condition may have deteriorated and adherence may have decreased, so albumin of $3 \mathrm{~g} / \mathrm{dL}$ or less was used as the standard.

The resulting significant variables were entered into the multivariate analyses. Multivariate analyses including significant variables were performed. Significance levels for univariate and multivariate analyses were set at $p<0.2$ and $p<0.05$, respectively. Odds ratios (ORs) and $p$-values were computed for the variables in logistic regression models. All analyses were performed using SPSS version 24.0 (SPSS, Chicago, IL,

Table 1. Patients Demographics and Clinical Characteristics

\begin{tabular}{|c|c|c|}
\hline Characteristic & Total $(n=116)$ & No. of patients $(\%)$ \\
\hline Median age, years (range) & \multicolumn{2}{|c|}{$66.0(21-80)$} \\
\hline \multicolumn{3}{|l|}{ Gender } \\
\hline Male/Female & $66 / 50$ & $56.9 / 43.1$ \\
\hline \multicolumn{3}{|l|}{ ECOG performance status } \\
\hline $0 / 1 / 2$ & $69 / 43 / 4$ & $59.5 / 37.1 / 3.4$ \\
\hline \multicolumn{3}{|l|}{ Disease status } \\
\hline Unresectable/Recurrent & $87 / 29$ & $75.0 / 25.0$ \\
\hline \multicolumn{3}{|l|}{ Tumor histology } \\
\hline Differentiated/Undiffrentiated & $76 / 40$ & $65.5 / 34.5$ \\
\hline \multicolumn{3}{|l|}{ Metastatic site } \\
\hline Peritoneum & 64 & 55.2 \\
\hline Lymph nodes & 60 & 51.7 \\
\hline Liver & 24 & 20.7 \\
\hline Lung & 2 & 1.7 \\
\hline \multicolumn{3}{|l|}{ Metastatic site } \\
\hline $0 / 1 / 2 / \geqq 3$ & $4 / 62 / 44 / 6$ & $3.5 / 53.5 / 37.9 / 5.1$ \\
\hline \multicolumn{3}{|l|}{ Chemotherapy } \\
\hline SOX + Trastuzumab/SOX & $105 / 11$ & $90.5 / 9.5$ \\
\hline \multicolumn{3}{|l|}{ Number of combined drugs } \\
\hline $0-4 / \geqq 5$ & $95 / 21$ & $81.9 / 18.1$ \\
\hline
\end{tabular}

Abbreviations: ECOG, Eastern Cooperative Oncology Group.
U.S.A.).

\section{RESULTS}

Patients and Characteristics A total of 174 patients were identified from the outpatient pharmacy information systems of the Cancer Institute Hospital of the Japanese Foundation for Cancer Research. Patients with no data collected $(n=44)$, second-line SOX treatment $(n=9)$, continued SOX treatment from other hospitals $(n=3)$, and changed hospital in the first cycle $(n=2)$ were excluded. The demographic and clinical characteristics of the 116 patients analyzed are reported in Table 1 . The study included 116 patients, with a median age of 66.0 years (range: $21-80$ years), and 66 male and 50 female patients. All patients were diagnosed with metastatic gastric cancer. Six patients $(5.1 \%)$ had $\geq 3$ metastatic sites, 44 patients $(37.9 \%)$ had 2 metastatic sites, and 62 patients $(53.5 \%)$ had 1 metastatic site. Ninety-five patients $(81.9 \%)$ were taking 4 or fewer combined drugs, and 21 patients (18.1\%) were taking 5 or more combined drugs.

Adherence Rate Figure 2 shows the S-1 adherence rate on SOX treatment determined using the pill count by the pharmaceutical outpatient clinic. The median adherence rate was $92.8 \%(n=116)$ in the first cycle, $91.0 \%(n=110)$ in the second cycle, and $90.5 \%(n=40)$ in the seventh cycle. The median relative dose intensity of S-1 was $84.6 \%$.

Factors Reducing S-1 Adherence in SOX Treatment In total, non-adherence was seen in 1095 instances during cycles $1-7$. The most common reasons for non-adherence were nausea/vomiting (43.7\%, 479 instances), diarrhea (20.8\%, 228 instances), missed dose (11.8\%, 129 instances), and fever (8.1\%, 89 instances) (Fig. 3).

Multivariate Model of Factors Associated with S-1 Adherence in SOX Treatment Results of multivariate analysis

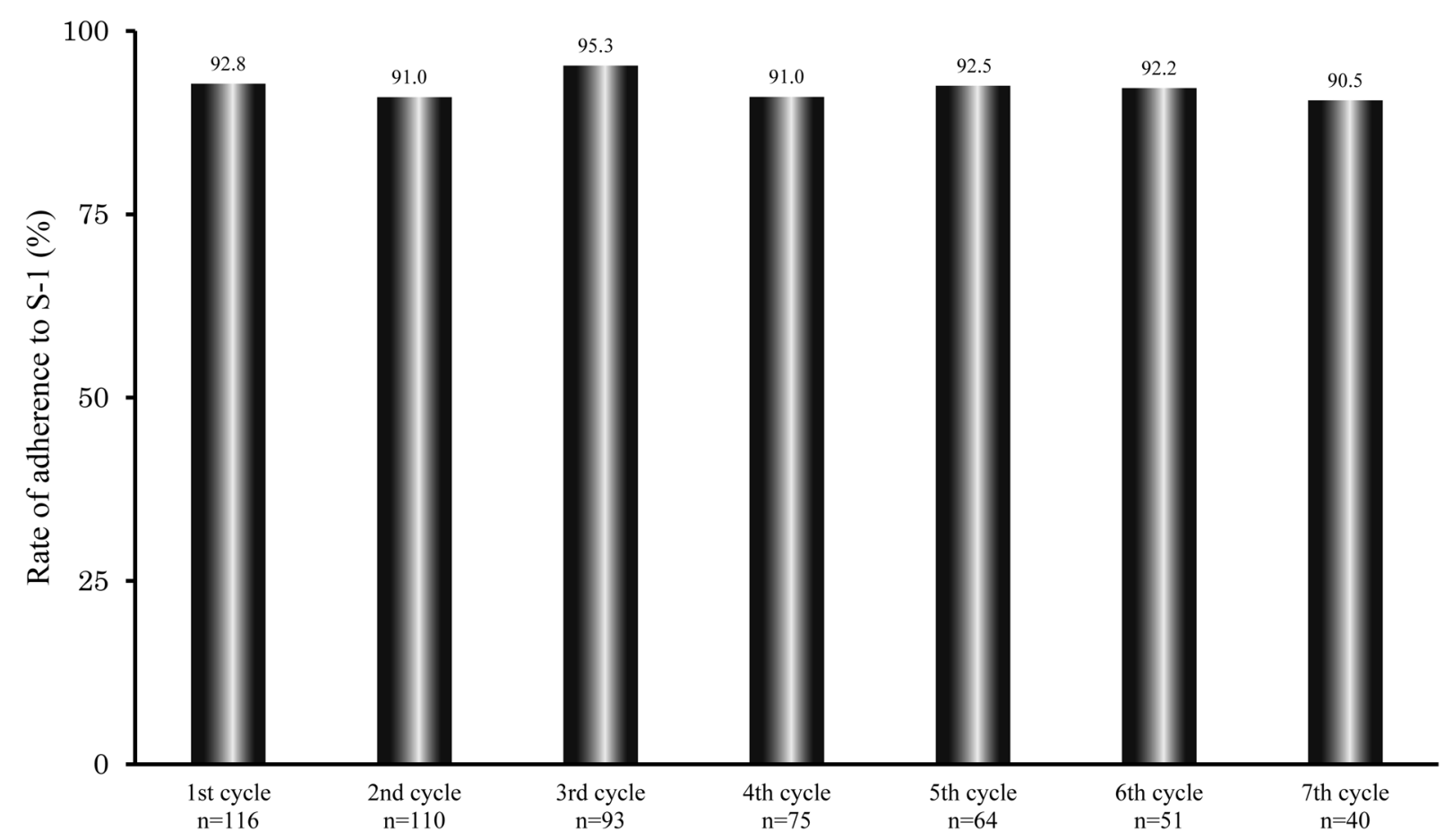

Fig. 2. S-1 Adherence Rate in SOX Treatment with Metastatic Gastric Cancer during Cycles 1-7

Adherence to S-1 was assessed using pill counts at the pharmaceutical outpatient clinic. 


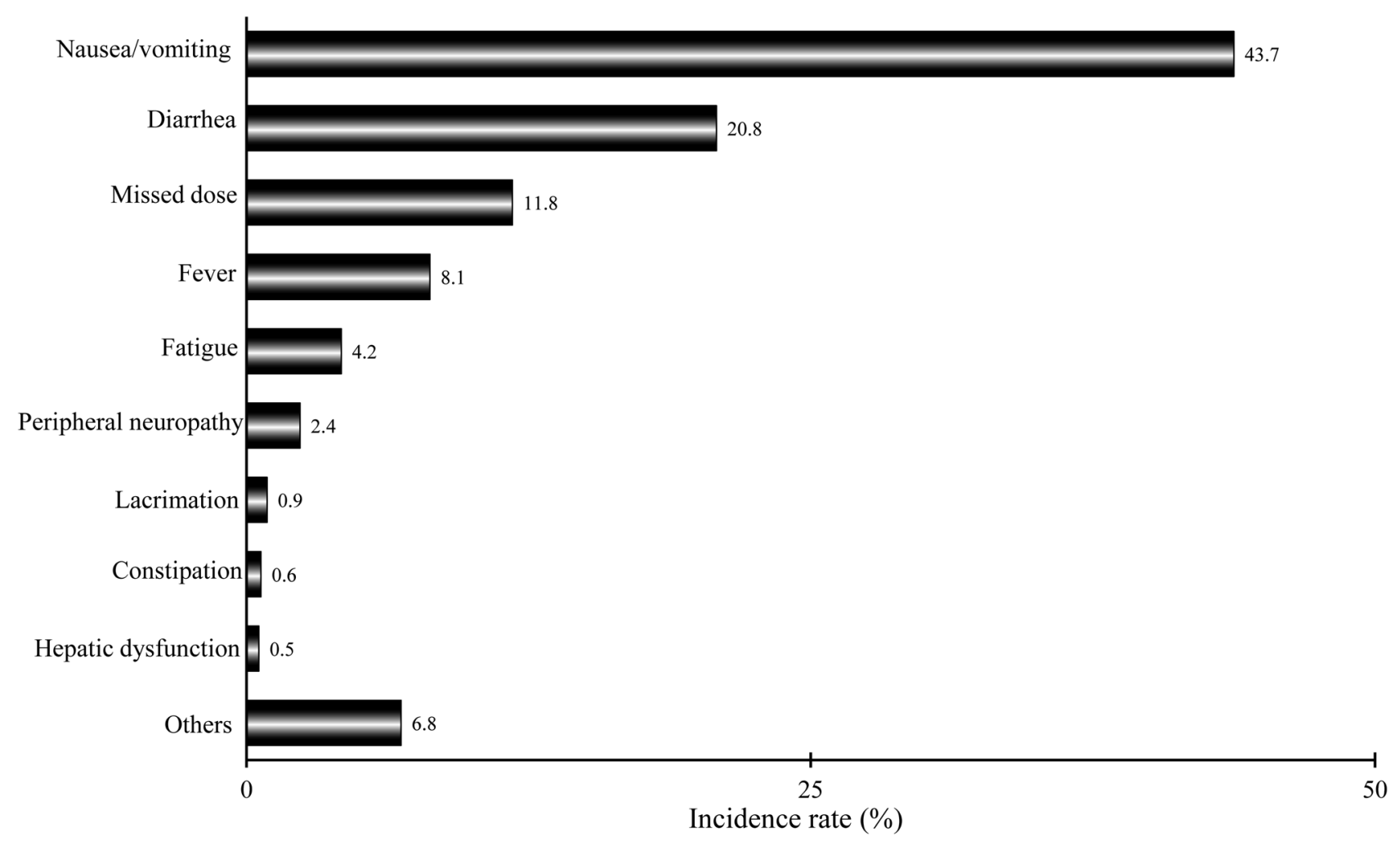

Fig. 3. Factors Reducing Adherence to S-1 on SOX Treatment during Cycles $1-7(n=1095)$

Others factors are stomatitis, dizziness, hiccup, etc.

Table 2. Logistic Regression Analysis of Optimal Adherence to Clinical Factors in Adherence Rate $\geqq 95 \%$ in the First Cycle

\begin{tabular}{|c|c|c|c|c|c|}
\hline \multirow{2}{*}{ Variables } & \multirow{2}{*}{ Category } & \multicolumn{2}{|c|}{ Univariate analysis } & \multicolumn{2}{|c|}{ Multivariate analysis } \\
\hline & & OR $(95 \% \mathrm{Cl})$ & $p$-Value & OR $(95 \% \mathrm{Cl})$ & $p$-Value \\
\hline Age & $\geq 70$ years old $($ ref. $<70)$ & $1.52(0.65-3.54)$ & 0.38 & & \\
\hline Sex & Female (ref. male) & $0.60(0.25-1.40)$ & 0.29 & & \\
\hline ECOG performance status & 2 (ref. 0,1$)$ & $0.273(0.36-20.27)$ & 0.30 & & \\
\hline Living status & Living alone (ref. with family) & $1.57(0.42-5.78)$ & 0.35 & & \\
\hline Creatinine clearance & $\leq 60 \mathrm{~mL} / \mathrm{min}($ ref. $>60 \mathrm{~mL} / \mathrm{min})$ & $1.55(0.62-3.84)$ & 0.47 & & \\
\hline Number of combined drugs & $\geq 5($ ref. $0-4)$ & $2.50(1.04-6.03)$ & $0.03 *$ & $2.50(1.04-6.03)$ & 0.04 \\
\hline Marriage & Unmarried (ref. married) & $0.40(0.11-1.50)$ & 0.18 & & \\
\hline Total gastrectomy & Yes (ref. No) & $1.66(0.62-4.46)$ & 0.42 & & \\
\hline Disease status & Unresectable (ref. resectable) & $0.79(0.31-2.00)$ & 0.81 & & \\
\hline ALB & $<3 \mathrm{~g} / \mathrm{dL}($ ref. $\geq 3 \mathrm{~g} / \mathrm{dL})$ & $0.76(0.19-2.98)$ & 0.49 & & \\
\hline Trastuzumab & Yes (ref. No) & $2.40(0.68-8.52)$ & 0.14 & & \\
\hline Oxaliplatin dose (first cycle) & $130 \mathrm{mg} / \mathrm{m}^{2}$ (ref. $100 \mathrm{mg} / \mathrm{m}^{2}$ ) & $0.66(0.26-1.67)$ & 0.50 & & \\
\hline
\end{tabular}

ECOG, Eastern Cooperative Oncology Group. ${ }^{*} p<0.05$.

are shown in Table 2. Univariate analyses identified number of combined drugs $(\geq 5)$ as a variable significantly associated with non-adherence $(p=0.04)$. When variables were selected among these 12 factors by the stepwise method, and logistic regression was performed using the most appropriate regression equation, a significant association was detected with 1 factor, the number of combined drugs $(\geq 5)(\mathrm{OR}=2.50 ; 95 \%$ confidence interval (CI), 1.04-6.03; $p=0.04)$.

\section{DISCUSSION}

This study showed the S-1 adherence rate course-by-course in SOX treatment and the factors reducing adherence by pill counts in the Pharmaceutical Outpatient Clinic. Adherence to S-1 was about $90 \%$ from courses 1 to 7 , and the reason for reduced adherence was mainly the major side effects of S-1 and oxaliplatin, such as nausea/vomiting and diarrhea. Furthermore, it was found that use of 5 or more combined drugs before the start of chemotherapy was the patient background factor that decreased adherence, which is useful real-world data.

This report is the first to demonstrate that the number of combined drugs $(\geq 5)$ was a significant risk factor for decreased adherence to S-1 in SOX treatment. Patients taking 5 or more combined drugs before SOX treatment are anxious about using supportive care medications (e.g., metoclopramide 
during nausea, loperamide during diarrhea). As a result, we considered that supportive care medications cannot be used properly and S-1 cannot be taken orally. A previous study reported that lean body-mass $\operatorname{loss}^{17)}$ and age over 65 years ${ }^{18)}$ were important risk factors for decreased adherence to adjuvant chemotherapy with S-1 in gastric cancer. In addition, considering the likelihood of side effects, adverse events, such as neutropenia and nausea/vomiting, were more frequently observed in female patients than in male patients in SOX treatment. ${ }^{19)}$ A postmarketing survey of S-1 involving 3294 patients with advanced gastric cancer identified demonstrated an association between the incidence of Grade 3 or worse hematological toxicity and renal function. ${ }^{20)}$ The incidence of nausea was slightly higher in patients who had undergone total gastrectomy than distal gastrectomy for capecitabine plus oxaliplatin for gastric cancer. ${ }^{21)}$ Based on the above, risk factors for decreased adherence to S-1 were female, impaired renal function, and total gastrectomy. In the present study, age, sex, renal function, and operative procedure were not factors that decreased S-1 adherence in SOX treatment. Age was 70 years and older, and the creatinine clearance was $60 \mathrm{~mL} / \mathrm{min}$ or less, so there appeared to be no significant difference. In addition, there were no significant differences in sex and operative method even in previous studies. ${ }^{19,21)}$ The present study focused on S-1 adherence and identified a novel risk factor from the viewpoint of combined drugs. Although the number of combined drugs increases as the number of pathological conditions increases, it is necessary to evaluate the necessity of drugs that are prescribed blindly (stomach drugs, intestinal regulators) and discontinue them.

Gastrointestinal toxicity such as nausea and vomiting was the most frequent reason for nonadherence to S-1 in SOX treatment. This is a side effect that seems to occur despite preventive measures. Even if explained to the patient in advance, there are cases in which S-1 is discontinued because of side effects. Healthcare professionals need to repeatedly instruct patients to take S-1 continuously even with supportive care. This study identified the factors that decrease adherence to $\mathrm{S}-1$. This is valuable information in actual clinical practice, and it is necessary to manage side effects by reducing the treatment intensity of chemotherapy.

A previous report on adjuvant S-1 chemotherapy for gastric cancer showed continuation rates at 12 months of 58.7 to $81.5 \%,{ }^{22)}$ and another retrospective study showed relative dose intensities of adjuvant S-1 of 82.9 to $84.7 \%{ }^{23)}$ This is the first report of adherence to S-1 in SOX treatment evaluated by pill counts. In the present study, the median relative dose intensity of S-1 was $84.6 \%$ during cycles $1-7$, and the median adherence rate was $92.8 \%$ in the first cycle and $90.5 \%(n=40)$ in the seventh cycle. Adherence to oral anticancer drugs tends to be higher than that to drugs for lifestyle-related diseases (hypertension, hyperlipidemia, etc.), but it was found that the adherence rate was the same as that to capecitabine ${ }^{12}$ (the median adherence rate was $94.0 \%$ ) and trifluridine plus tipiracil ${ }^{13)}$ (the adherence rates were 95.0\%). In addition, the relative dose intensity of S-1 in SOX treatment was $78.7 \%$ in previous studies, ${ }^{4}$ which is almost the same as in the present study, and is not particularly low. Adherence to S-1 of $90 \%$ or more is considered to be due to the effect of meticulous care in the pharmaceutical outpatient clinic by pharmacists and doctors. In addition to prescribing oral anticancer drugs, it is necessary to establish a system like a pharmaceutical outpatient clinic, and involvement of pharmacists ${ }^{24)}$ is required.

The present study had several limitations. First, the limitations include its retrospective nature and small sample size from a single institute. However, whereas other studies have evaluated adherence to many anticancer drugs, this study is valuable because it focused on S-1 in SOX treatment. Second, the present study assessed adherence to S-1 using pill counts. Medication event monitoring systems have been reported to be highly objective in assessing oral drug adherence. ${ }^{25)}$ However, the method of conducting patient interviews and pill counts that was used is also one of the objective evaluations and is considered provide valuable data.

Oral anticancer drugs are used as standard therapy for many cancer types. Oral anti-cancer drugs, unlike injectable drugs, are effective only when taken properly at home. To increase adherence to oral anticancer drugs in clinical practice, it is useful to consider the reasons for non-adherence to S-1 in this study. Pharmacist counselling with cancer patients is needed. ${ }^{26)}$ Specifically, use of five or more combined drugs was identified as a risk factor. Patients with advanced gastric cancer who are eligible for SOX treatment often use gastric drugs, iron preparations, and analgesics in combination. Claxton et al. found that adherence was inversely proportional to frequency of dose. ${ }^{27)}$ The adherence was inversely proportional to frequency of dose. For gastric drugs, it is necessary to take measures such as a proton pump inhibitor once a day rather than a gastric mucosa protective agent taken three times a day, and for iron drugs, the usage should be once a day. It is important to take measures to discontinue prescriptions for drugs that are not needed.

Conflict of Interest K. Yamaguchi has received speaking honorariums from Taiho Pharmaceutical, Chugai Pharmaceutical, Merck Serono, Takeda Pharmaceutical, Yakult, Bayer, Ono Pharmaceutical, Eli Lilly, Sanofi and Bristol-Myers Squibb, and has received research Grants from MSD, Ono Pharmaceutical, Sumitomo Dainippon Pharma, Taiho Pharmaceutical, Daiichi Sankyo, Eli Lilly, Gilead Sciences, and Yakult. Also, he has served in a consulting or advisory role for BristolMyers Squibb. The other authors declare that they have no conflict of interest.

\section{REFERENCES}

1) Ferlay J, Soerjomataram I, Dikshit R, Eser S, Mathers C, Rebelo M, Parkin DM, Forman D, Bray F. Cancer incidence and mortality worldwide: sources, methods and major patterns in GLOBOCAN 2012. Int. J. Cancer, 136, E359-E386 (2015).

2) Koizumi W, Narahara H, Hara $T$, Takagane A, Akiya $T$, Takagi M, Miyashita K, Nishizaki T, Kobayashi O, Takiyama W, Toh Y, Nagaie T, Takagi S, Yamamura Y, Yanaoka K, Orita H, Takeuch M. S-1 plus cisplatin versus S-1 alone for first-line treatment of advanced gastric cancer (SPIRITS trial): a phase III trial. Lancet Oncol., 9, 215-221 (2008).

3) Cunningham $D$, Starling $N$, Rao $S$, Iveson $T$, Nicolson $M$, Coxon F, Middleton G, Daniel F, Oates J, Norman AR. Capecitabine and oxaliplatin for advanced esophagogastric cancer. N. Engl. J. Med., 358, 36-46 (2008).

4) Yamada Y, Higuchi K, Nishikawa K, et al. Phase III study comparing oxalipalatin plus S-1 with cisplatin plus S-1 in chemotherapy-naïve patients with advanced gastric cancer. Ann. Oncol., 26 
141-148 (2015)

5) Shirasaka T, Nakano K, Takechi T, Satake H, Uchida J, Fujioka A, Saito H, Okabe H, Oyama K, Takeda S, Unemi N, Fukushima M. Antitumor activity of $1 \mathrm{M}$ tegafur-0.4 M 5-chloro-2,4-dihydroxypyridine-1 M potassium oxonate (S-1) against human colon carcinoma orthotopically implanted into nude rats. Cancer Res., 56, 2602-2606 (1996).

6) Kubota T. The role of S-1 in the treatment of gastric cancer. $B r . J$. Cancer, 98, 1301-1304 (2008).

7) Timmers L, Boons CC, Kropff F, van de Ven PM, Swart EL, Smit EF, Zweegman S, Kroep JR, Timmer-Bonte JN, Boven E, Hugtenburg JG. Adherence and patients' experiences with the use of oral anticancer agents. Acta Oncol., 53, 259-267 (2014).

8) Partridge AH, Avorn J, Wang PS, Winer EP. Adherence to therapy with oral antineoplastic agents. J. Natl. Cancer Inst., 94, 652-661 (2002).

9) Osterberg L, Blaschke T. Adherence to medication. N. Engl. $J$. Med., 353, 487-497 (2005).

10) WHO. Adherence to Long-Term Therapies: Section III, Chapter VIII Cancer, WHO (2003).

11) Hryniuk WM, Goodyear M. The calculation of received dose intensity. J. Clin. Oncol., 8, 1935-1937 (1990).

12) Kawakami K, Yokokawa $T$, Kobayashi K, Sugisaki T, Suzuki K, Suenaga M, Yamaguchi K, Inoue A, Machida Y, Yamaguchi T, Hama T. Self-reported adherence to capecitabine on XELOX treatment as adjuvant therapy for colorectal cancer. Oncol. Res., 25, 1625-1631 (2017).

13) Sugita K, Kawakami K, Yokokawa $T$, Sugisaki T, Takiguchi T, Aoyama T, Suzuki K, Suenaga M, Yamaguchi K, Inoue A, Machida Y, Yamaguchi T, Hama T. Self-reported adherence to trifluridine and tipiracil hydrochloride for metastatic colorectal cancer: a retrospective cohort study. Oncology, 91, 224-230 (2016).

14) Winterhalder R, Hoesli P, Delmore G, Pederiva S, Bressoud A, Hermann F, von Moos R. Self-reported compliance with capecitabine: findings from a prospective cohort analysis. Oncology, 80, 29-33 (2011).

15) Kawakami K, Nakamoto E, Yokokawa T, Sugita K, Mae Y, Hagino A, Suenaga M, Mizunuma N, Oniyama S, Machida Y, Yamaguchi T, Hama T. Patients' self-reported adherence to capecitabine on XELOX treatment in metastatic colorectal cancer: findings from a retrospective cohort analysis. Patient Prefer. Adherence, 9, 561-567 (2015).

16) Kojima T, Akishita M, Kameyama Y, Yamaguchi K, Yamamoto $\mathrm{H}$, Eto M, Ouchi Y. High risk of adverse drug reactions in elderly patients taking six or more drugs: analysis of inpatient database. Geriatr. Gerontol. Int., 12, 761-762 (2012).

17) Aoyama $T$, Kawabe $T$, Fujikawa $H$, Hayashi $T$, Yamada $T$, Tsuchida K, Yukawa N, Oshima T, Rino Y, Masuda M, Ogata T, Cho H, Yo- shikawa T. Loss of lean body mass as an independent risk factor for continuation of S-1 adjuvant chemotherapy for gastric cancer. Ann. Surg. Oncol., 22, 2560-2566 (2015).

18) Yamashita K, Kurokawa Y, Yamamoto K, Hirota M, Kawabata R, Mikami J, Masuzawa T, Takiguchi S, Mori M, Doki Y. Risk factors for poor compliance with adjuvant S-1 chemotherapy for gastric cancer: a multicenter retrospective study. Ann. Surg. Oncol., 24, 2639-2645 (2017).

19) Yamada Y, Koizumi W, Nishikawa K, Gotoh M, Fuse N, Sugimoto $\mathrm{N}$, Nishina T, Amagai $\mathrm{K}$, Chin $\mathrm{K}$, Niwa $\mathrm{Y}$, Tsuji A, Imamura $\mathrm{H}$, Tsuda M, Yasui H, Fujii H, Yamaguchi K, Yasui H, Hironaka S, Shimada K, Hyodo I. Sex differences in the safety of S-1 plus oxaliplatin and S-1 plus cisplatin for patients with metastatic gastric cancer. Cancer Sci., 110, 2875-2883 (2019).

20) Nagashima F, Ohtsu A, Yoshida S, Ito K. Japanese nationwide postmarketing survey of S-1 in patients with advanced gastric cancer. Gastric Cancer, 8, 6-11 (2005).

21) Fuse $\mathrm{N}$, Bando $\mathrm{H}$, Chin $\mathrm{K}$, Ito $\mathrm{S}$, Yoshikawa T, Tsuburaya A, Terashima M, Kawashima Y, Fukunaga T, Gotoh M, Emi Y, Yoshida K, Oki E, Takahashi S, Kuriki H, Sato K, Sasako M. Adjuvant capecitabine plus oxaliplatin after D2 gastrectomy in Japanese patients with gastric cancer: a phase II study. Gastric Cancer, 20, 332-340 (2017)

22) Yamashita K, Kurokawa Y, Yamamoto K, Hirota M, Kawabata R, Mikami J, Masuzawa T, Takiguchi S, Mori M, Doki Y. Risk factors for poor compliance with adjuvant S-1 chemotherapy for gastric cancer: a multicenter retrospective study. Ann. Surg. Oncol., 24, 2639-2645 (2017).

23) Timmers L, Boons CCLM, Kropff F, van de Ven PM, Swart EL, Smit EF, Zweegman S, Kroep JR, Timmer-Bonte JNH, Boven E, Hugtenburg JG. Adherence and patients' experiences with the use of oral anticancer agents. Acta Oncol., 53, 259-267 (2014).

24) Imamura $M$, Ogawa $D$, Takatori $T$, Yamaguchi $M$, Takata $T$, Hada T, Ota Y, Uehara T. A retrospective study of the effects of oncology pharmacist participation in treatment on therapeutic outcomes and medical costs. Biol. Pharm. Bull., 40, 1956-1962 (2017).

25) van den Boogaard J, Lyimo RA, Boeree MJ, Kibiki GS, Aarnoutse RE. Electronic monitoring of treatment adherence and validation of alternative adherence measures in tuberculosis patients: a pilot study. Bull. World Health Organ., 89, 632-639 (2011).

26) Kawaguchi T, Azuma $K$, Yamaguchi $T$, Iwase $S$, Matsunaga $T$, Yamada K, Miyamatsu H, Takeuchi H, Kohno N, Akashi T, Unezaki S. Preferences for pharmacist counselling in patients with breast cancer: a discrete choice experiment. Biol. Pharm. Bull., 37, 1795-1802 (2014).

27) Claxton AJ, Cramer J, Pierce C. A systematic review of the associations between dose regimens and medication compliance. Clin. Ther., 23, 1296-1310 (2001). 